\title{
Challenges of Nurses in the Caring Process of Patients with Coronavirus Disease 2019 in Iran: A Qualitative Study
}

\author{
Simintaj Sharififar ${ }^{1}$, Maryam Moradi ${ }^{1}$ and Milad Ahmadi Marzaleh ${ }^{2, *}$ \\ ${ }^{1}$ Department of Health in Disasters and Emergencies, School of Nursing, AJA University of Medical Sciences, Tehran, Iran \\ 2 Department of Health in Disasters and Emergencies, School of Management and Medical Informatics, Shiraz University of Medical Sciences, Shiraz, Iran
}

* Corresponding author: Milad Ahmadi Marzaleh, Department of Health in Disasters and Emergencies, School of Management and Medical Informatics, Shiraz University of Medical Sciences, Shiraz, Iran. Tel: +987132340774; Fax: +987132340039; Email: miladahmadimarzaleh@yahoo.com

Received 2021 February 03; Revised 2021 February 28; Accepted 2021 April 18.

\begin{abstract}
Background: The illness caused by the novel coronavirus is an emerging disease, and for this reason, the international community is encountered with its unknown nature, such as clinical features, treatments, and long-term complications. Experience suggests that humans are affected by the psychological and social effects of epidemics and pandemics of infectious diseases. Accordingly, it is a new experience to care for patients with this disease that has to be more explored.

Objectives: The present study aimed to detect the challenges with which nurses are faced in the process of caring for patients with coronavirus disease 2019 (COVID-19) in Iran using a qualitative method.

Methods: This qualitative study utilized in-depth semi-structured interviews with 19 key informants selected via targeted sampling. The obtained data were analyzed by thematic analysis to identify the challenges to which the nurses have encountered in the process of caring for patients with COVID-19 in Iran.

Results: Challenges of the nurses in the caring process for patients with COVID-19 are listed in five categories of stress and psychological issues, equipment-related challenges, increased events of errors, medication and treatment problems, and hospital management. It should be mentioned that at the same time, 35 other subcategories were also identified in this study.

Conclusion: Since nurses are at the forefront in fighting the COVID-19, there must be plans to increase their preparedness. Deficiencies in health systems, including equipment, medicine, and personal protective gear should also be addressed so that nurses can better respond to these incidents. Psychological and mental support is a key component of the nurses' appropriate response; therefore, along with nurses' responses, individuals, groups, and family psychological supports should be facilitated to increase performance and effectiveness in response to COVID-19.

Keywords: COVID-19, Iran, Novel Coronavirus, Nurses, Preparedness, Quality
\end{abstract}

\section{Background}

In December 2019, some unexplained cases of pneumonia were reported in Wuhan, Hubei Province, China. Epidemiological findings showed severe human-to-human transmission. Following that, the World Health Organization (WHO) named it Coronavirus Disease 2019 (COVID-19) (1). The virus is highly contagious during incubation, compared to viruses, such as SARS and Ebola, as it has asymptomatic infections transmitted through breathing droplets, contact, and airborne particles (2). Due to its rapid spread, severe infection, mortality, and unknown drug treatment, it poses a great threat to human life and health. As of April 3, 2020 , the number of patients worldwide exceeded two million and about 120,000 people lost their lives (3). In addition to affecting people's physiques, the disease has an enormous effect on the mental health of the general public with individuals demonstrating varying degrees of social psychological problems, such as fear and anxiety (4). However, the emergence of SARS in China had previously caused serious concern, fear, as well as severe psychological and social disorders $(5,6)$.
Hospitals and health center staff are directly affected by the incidents and crises $(7,8)$. In biological crises, this response is much more important than that of other crises since the likelihood of pathogen transmission to the staff in health care centers is higher than that to the other members of society $(9,10)$. The disease caused by the new COVID-19 virus is a major challenge for the staff at the forefront of the challenge, especially nurses, due to its novelty, lack of definitive treatment, as well as lack of drug prophylaxis and vaccination. Nurses are usually the first and only health experts to feel the need for health care in this field. Armed Forces Hospitals are among the main respondents to the COVID-19 crisis in the country. Nurses in these centers are active in a range of care and management roles (11). An explanation of the challenges of this group of health workers in response to this emerging disease has played a significant role in discovering the themes of patient care and the concept of care for patients with COVID-19; accordingly, it is essential to remove the challenges of increasing health care demand. The use of the experiences of this group as the key members of the treatment team in the care of patients with COVID- 19 can prevent similar problems and 
eliminate confusion in potential future events.

COVID-19 is a new disease, and for this, the international community is faced with the unknown nature of its dimensions, such as clinical signs and symptoms, treatments, and long-term side effects. Experience shows the psychological and social effects on humans because of epidemics and pandemics of infectious diseases. It is a new experience to take care of patients with this disease, which has to be explored. Nurses' perception of the status of care for patients with the disease is complex and probably depends on the circumstances associated with the conditions in their cultural and social contexts. Although no study has been published in this field in the world, it can be stated that even if there is such research in other countries, the issue cannot be generalizable to Iranian nurses. Therefore, this qualitative study was conducted to explain the experiences of frontline nurses after the discovery of challenges to care for these patients.

\section{Objectives}

The present study aimed to detect the challenges with which nurses are faced in the process of caring for patients with coronavirus disease 2019 (COVID19) in Iran using a qualitative method.

\section{Methods}

This study was performed using a descriptive qualitative method from February 20 to April 18, 2020, which was the peak period of the COVID-19 epidemic in Iran. The information was collected through interviews with key experts and informants. Data were analyzed using thematic analysis to express the challenges of nurses in the caring process for patients with COVID-19 in Iran.

\subsection{Participants}

In-depth and semi-structured interviews were conducted with experts and nurses, and qualitative data analysis was performed using a thematic analysis procedure. The inclusion criteria were at least having a bachelor's degree, work experience in caring for patients with COVID-19, or managerial work and related research as well as a willingness to take part in the research. If any of the experts were not willing to participate in the study before or during the interview, they could withdraw from the study at any time.

It is worth mentioning that the study participants were all residents of Tehran province, Iran. The sampling method was purposive, and people who had lots of information were identified in this study. Interviews were conducted with front-line nurses (i.e., those who were working in the special respiratory, emergency, and private wards of COVID19 in the designated hospitals for these patients).
Subsequently, the interviews were conducted after explaining the objectives and significance of the research, as well as obtaining their consent. The researcher then referred to them at an appropriate time. People's names were not included in the demographic forms, and numbers (i.e., \#1, \#2,...) were used to name the individuals instead.

\subsection{Data Collection}

In-depth and semi-structured individual interviews were conducted with key informants. The question of the interview was open-ended, and the semi-structured interview was such that experts and key experts were asked to speak about the challenges of nurses in the process of caring for patients with COVID-19. The semistructured interview started by asking some questions, such as: "What challenges did you have in providing services to patients with COVID-19?", and follow-up questions, such as: "How?", "Why?", "Can you explain more about ...?" were also asked during the interview. It should be mentioned that the questions might be changed during the research course due to the analysis of the initial interviews.

Moreover, several other aspects of the interviewee's comments that were raised during the conversation were investigated when speaking about the topic under discussion with another followup/probing question asked. In total, 19 interviews were conducted with 19 experts. Finally, the interviews ended with data saturation. Each interview lasted about 30-75 min. The interviews were conducted by the researcher, and to avoid possible errors in recording the specialists' voices, the interviews were recorded by two voice recorders and were immediately released after the performance.

\subsection{Data Analysis}

Along with data collection, the analysis was also performed. All interviews were written word by word immediately after recording, and the researchers also took notes during the interview. Thematic analysis was used to analyze the data.

The six-step thematic analysis included: 1) Familiarization with the data (and extraction of concepts from the items), 2) Creation of primary codes (or assigning a code to each of the concepts and their main and sub-categories), 3) Category search, 4) Main and subcategory review: At this stage, the code, categories, and subcategories were reviewed about the data set, 5) Definition and naming of categories and subcategories, and 6) Preparation of a report (12). If necessary, interviews were provided to individuals to get the errors corrected or contents added. At the end of the interview, the texts were manually analyzed; moreover, the codes and categories were grouped with the categories and subcategories extracted. After analyzing the text of the interviews, 492 codes were finally extracted, which were categorized into 5 categories and 35 
subcategories by deleting repetitive codes and reanalyzing them.

\subsection{Rigor}

To ensure the accuracy and precision of qualitative data, Guba and Lincoln criteria (13), which include trustworthiness, transformability, dependability, and confirmability were used in this study. The interviews were analyzed several times. The text of the interviews was provided to the participants to correct the possible errors. Different coding procedures were also utilized in this study, and the interviews were analyzed with the help of a qualitative study specialist.

\subsection{Ethical Considerations}

The present study was approved by the Ethics Committee in biomedical research of AJA University of Medical Sciences, Tehran, Iran (IR.AJAUMS. REC.1399.056). After obtaining the necessary permissions from the Deputy for Research at the School of Nursing and a letter of confirmation from the AJA University of Medical Sciences, the researchers presented them to the research samples and introduced themselves to the participants, explained the objectives of the research, and reassured them that all the recorded issues would be kept confidential. Afterward, the participants who were willing to participate in the study were selected, and they were also assured that they could withdraw from the interview process at any stage. Other ethical considerations included: 1) Obtaining written consent from the experts, 2) Reassuring the participants that they will be provided with the results of the study if they wished 3) Observing the ethical considerations in terms of confidentiality of the data 4) Acknowledging and appreciating all the people who cooperated in the research, and 5) Obtaining approval from the ethics committee.

\section{Results}

The majority of the participants were female $(n=12)$. Moreover, the mean age and work experience of the respondents were obtained at 31.47 and 11.42 years, respectively. The demographic and occupational characteristics of the participants are shown in Table 1.

Table 1. Demographic and Occupational Characteristics of the Participants

\begin{tabular}{llcc}
\hline Characteristics & & No. & (\%) \\
\hline \multirow{2}{*}{ Gender } & Male & 7 & 36.84 \\
& Female & 12 & 63.15 \\
\multirow{2}{*}{ Marital status } & Single & 8 & 42.10 \\
& Married & 11 & 57.89 \\
\hline \multirow{2}{*}{ Education level } & Bachelor's degree & 17 & 89.47 \\
& Master's degree & 2 & 10.52 \\
\multirow{2}{*}{ Occupation } & Nurse at wards & 16 & 84.21 \\
& Ward supervisor & 3 & 15.78 \\
\hline
\end{tabular}

The challenges to which the nurses were faced in the process of caring for patients with COVID-19 in Iran are divided into five main categories, including stress and psychological issues; equipment-related challenges; increased error events; treatment and medication problems; and hospital management. Table 2 tabulates all these subcategories.

A summary of what the experts said is as follows:

A 35-year-old female nurse said: "...There was a pervasive sense of fear everywhere that the novel coronavirus kills people. We had heard that those who have underlying diseases would easily die of the disease, but we had reports of casualties having no underlying diseases including the elderly, athletes, young, and a group of nurses who had died of it before people were hospitalized here. Well. I am referring to Narjes, the Gilani nurse who died. I was shocked and scared. There was a sense of fear in the beginning".

A 30-year-old female nurse stated: “...My parents were very concerned about me, telling me not to expose myself to the disease. I told them I was wearing protective gear. Upon seeing me in the gear, my father sat down and cried a lot. It was not such that they would ever deter me from working there. On the other hand, my sister was saying, "this is what she has opted for". There will be good and bad days. My husband also said, "I could not escape the responsibility since I have chosen it and it's not optional to leave it".

A 28-year-old female nurse stated: “...Equipment began to arrive during my second shift and a pair of clothes was given for each working shift. I think it is better to give us two pairs, one before and the other after the resting time. It's really hard to work with such gears, though it is a type of assurance for the personnel. However, the guys here have no faith in the masks, which were distributed. They keep complaining that they are not $N 95$ masks. There was a concern about these masks whether to use them or not".

A 38-year-old- female nurse said: “...For the personnel, what was more important was individual protective equipment. If the hospital facilities allowed, several pairs of clothes and equipment would be prepared for the individual protective gear. From a spiritual point of view, it was also important to support the nurses. In the center we were in, there were some benefits specific to the personnel. I think the same individual protective equipment should be considered for the patients as they are involved with the diseases though the equipment was little".

A 39-year-old male nurse said: “...It took us a long time to get the medicines that we wanted to give the patients. Apart from the medicines, they needed other things. The services we delivered were timeconsuming. In the wards, the patients were connected to the ventilator, for example, it was very difficult to control the patient's fever. We brought the patient's 


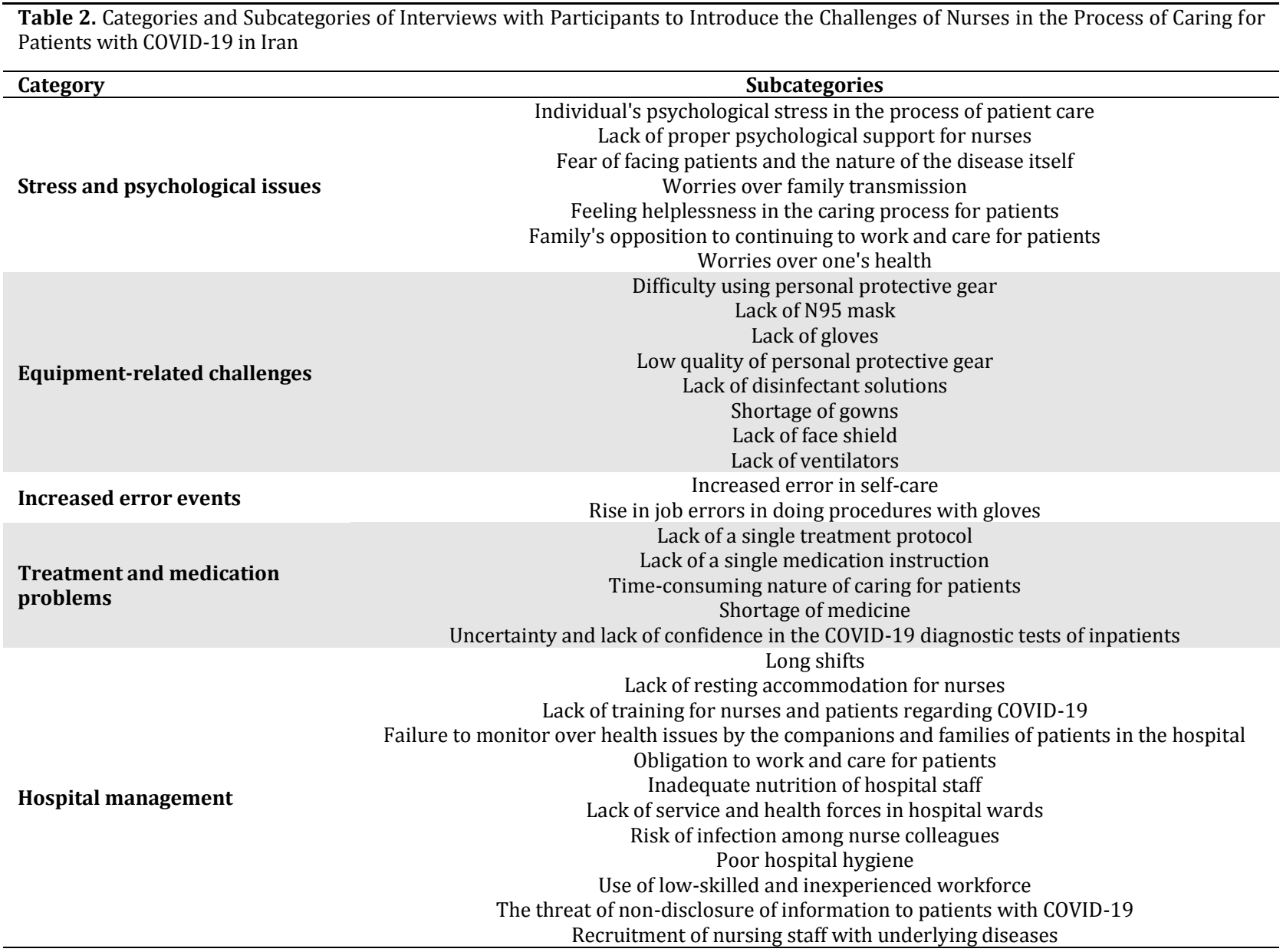

fever down; an hour later, then it rose to 40. Now, I do not know whether the virus has weakened or not, but now the patients are much better as the disease is less severe".

\section{Discussion}

This study is the first qualitative study on the challenges of nurses in the process of caring for patients with COVID-19 in Iran. No such qualitative study was found in this regard in Iran or across the world. This study provides experts and readers with a comprehensive perception of the nurses' challenges in the process of caring for patients with COVID-19. Given the fact that the key experts in this study were from a wide range of occupations, the challenges in these events were examined from different perspectives. According to the key experts, there are many challenges in the process of caring for patients with COVID-19; however, special planning and coordination are required in this regard. To respond properly to the virus, all hospital staff, especially nurses, must work together in a coordinated manner.

Personal protective equipment should be provided to patients before the nurses arrive. The quality of the protective equipment for the person plays a vital role in the comfort of nurses and the facilitation of the care process. Furthermore, one can assure complete protection by providing appropriate training for nurses, patients, and their companions. Nurses with stress and anxiety about COVID-19 should get psychological counseling. The presence of a psychologist in different wards of a hospital can reduce the nurses' stress and worries. Hospital management can improve the treatment process of patients by providing various equipment to nurses, trusting them, and creating an atmosphere of friendship and closeness amongst the staff.

In their study, Huang et al. investigated the emotional responses and coping strategies of nurses and nursing students during the prevalence of COVID-19. The results showed that women suffered more anxiety and fear, compared to men. In addition, participants in cities had more anxiety and fear than those in rural areas. Nurses working with patients that are affected by COVID-19 were suffering from anxiety and rage. Compared to nursing students, nurses formed more appropriate emotional responses to the problem (4). Female nurses are more prone to depression and stress due to their mental, psychological, and physiological conditions (14). Therefore, the presence of psychologists in hospitals can be helpful.

In their study entitled "Nursing Team: Nursing Managers' Experience in Acute Care Conditions", Ferguson et al. showed that nursing experience 
amongst nursing managers team was described in three main categories of compatibility with the nursing team, nursing team achievements, and nursing team interventions. The results suggested that this team-centered approach was considered by managers, enabling the nursing staff to provide a variety of experiences and skills with greater safety under direct supervision (15). The promotion of the activities of the main team in the hospital is the key to speeding up the performance of the tasks and treatment of patients. Hospital management can improve this process by creating a team-center atmosphere.

Similarly, Terry Reb et al. conducted a study on the preparation for bio-terrorism in Missouri nurses and found that $82.3 \%$ of respondents believed that bioterrorism attacks would occur in the United States; however, $21.3 \%$ specified that this would possibly occur less than once. Moreover, $97 \%$ of nurses believed that bioterrorism attacks would culminate in dire consequences, and $60 \%$ of the respondents said they had not been trained for bioterrorism and had not participated in the exercises. In addition, $20 \%$ of the nurses said they were unaware of the content of the bio-terrorism response program. Nurses need to be aware and encouraged to participate in educational opportunities and exercises in bio-terrorism and disasters (16). Furthermore, they require to take part in various educational programs on COVID-19, which can make nurses more prepared to deal with the disease and patients.

According to a study performed by Considine on the preparing and understanding of emergency nurses for radiation, biological, and chemical events, one-third of participants reported restrictions on the use of personal protective gear, and only a few participants had experienced these events; however, $70.3 \%$ of the participants were already trained on the mentioned issues. There were significant differences in perception and adequacy of training. The results of this study showed that emergency nurses were eager to meet the Chemical, Biological, and Radiological challenges. They also had a positive view of understanding these events. As nurses become more aware, their tendency to work in these situations increases (17). Therefore, it is critical to raise their awareness and knowledge so that they can respond more appropriately during these events.

In another study by Young et al. entitled "Readiness for Nuclear, Chemical, and Biological Terrorism: Major Concerns and Nurses Preparation in the Future", it was shown that the major concern of the students was about themselves and their families. They were primarily concerned about the proper protection against all types of terrorist incidents and stated that they would not be willing to take care of the victims if they were not provided with adequate support. In general, students did not have a deep understanding of the disease nature caused by terrorism (18). Since nursing students are at the forefront of responding to biological disasters, training programs should be considered to improve their preparedness.

The results of a study carried out by Lam Kam Ki et al. in 2013 showed that concerns about health, executive and managerial problems, and attitudes toward professionalism were among the most important nursing problems (i.e., working with patients who have influenza). The flu also threatens the health of the nurse and that of their family. Improvements in planning, an increase in the nurses' knowledge and information, and allocation of personal protective equipment can be helpful in this regard (19). The findings of the mentioned study are consistent with those of the present study, and some of the described subcategories were similar to those in the present study. In the same vein, McMullan et al. found that $90 \%$ of nurses considered their job to treat and care for patients with flu. Nurses are concerned about the increase in work shifts and the possibility of developing an infection in themselves and their families. Meanwhile, nurses' stress had significantly increased (20).

Coronavirus-caused illness is an emerging disease; therefore, the international community is unaware of its dimensions, such as clinical features, treatments, and long-term complications. Past experiences suggest psychological and social effects on humans because of the epidemics and pandemics of infectious diseases. As a new experience, taking care of the patients with this disease has to be explored. Nurses need to be mentally and emotionally prepared to meet the challenges of increasing healthcare demands. The experiences gained by nurses as vital members of the treatment team in critical situations can be a promising way to solve and prevent many problems. Nurses' understanding of the care condition of people with COVID-19 is complex and may depend on circumstances and contexts associated with their cultural and social background.

The findings of this study have several implications: a) assigning specific training to prepare nurses to pay attention to patients; b) preparing and setting up comprehensive guidelines and instructions for the treatment of patients, and; c) equipping hospitals in response to biological emergencies.

\section{Conclusion}

Given that nurses are at the forefront of responding to COVID-19, there must be plans to increase their preparedness. Shortages in health systems, including equipment, medicine, and personal protective equipment should also be addressed so that nurses can have a better 
understanding of these incidents. Psychological and mental support is a key component in the nurses' appropriate response; accordingly, along with nurses' responses, individuals, groups, and family psychological supports should also be provided for further efficiency and effectiveness in response to COVID-19.

\section{Acknowledgments}

Hereby, the authorities and personnel of AJA University of Medical Sciences, Tehran, Iran, are greatly appreciated for paving the way for conducting this study.

\section{Footnotes}

Author's contributions: Simintaj Sharififar and Milad Ahmadi Marzaleh were responsible for the study conception and design. Simintaj Sharififar, Maryam Moradi, and Milad Ahmadi Marzaleh supervised the whole study. Simintaj Sharififar, Maryam Moradi, and Milad Ahmadi Marzaleh prepared the first draft of the manuscript. Simintaj Sharififar, Maryam Moradi, and Milad Ahmadi Marzaleh analyzed the results and supervised the study. All authors have read and approved the final manuscript.

Conflicts of Interest: The authors have no conflict of interest to declare.

Ethical Approval: All participants were informed about the research objectives, and written consent was obtained from them. Moreover, they were assured that their information would remain confidential. They were also informed about their right to withdraw from the study at any time they want.

Funding/Support: This project has partly been supported by a grant from AJA University of Medical Sciences, Tehran, Iran (IR.AJAUMS.REC.1399.056).

Financial Disclosure: None declared.

Informed Consent: Informed consent was obtained from all individual participants in the study.

\section{References}

1. World Health Organization. Clinical management of severe acute respiratory infection when novel coronavirus ( 2019$\mathrm{nCoV}$ ) infection is suspected: interim guidance. Geneva: World Health Organization; 2020.

2. Wang $Y$, Wang $Y$, Chen $Y$, Qin Q. Unique epidemiological and clinical features of the emerging 2019 novel coronavirus pneumonia (COVID-19) implicate special control measures. $J$ Med Virol. 2020;92(6):568-76. doi: 10.1002/jmv.25748. [PubMed: 32134116].
3. CDC COVID-19 Response Team. Severe outcomes among patients with coronavirus disease 2019 (COVID-19) - United States, February 12-March 16, 2020. MMWR Morb Mortal Wkly Rep. 2020;69(12):343-6. doi: 10.15585/mmwr.mm6912e2. [PubMed: 32214079].

4. Huang L, Lei W, Xu F, Liu H, Yu L. Emotional responses and coping strategies in nurses and nursing students during Covid19 outbreak: A comparative study. PLoS One. 2020;15(8): e0237303. doi: 10.1371/journal.pone.0237303. [PubMed: 32764825].

5. Chiu HF, Lam LC, Li S, Chiu E. SARS and psychogeriatrics: perspective and lessons from Hong Kong. Int J Geriatr Psychiatry. 2003;18(10):871-3. doi: 10.1002/gps.1003. [PubMed: 14533118].

6. Leung PC, Ooi EE. SARS war: combating the disease. Singapore: World Scientific; 2003.

7. Zaboli R, Tofighi S, Seyyedin S, Malmoon Z, Hoseini Shokuh S. Organizational vulnerability and management of clinical departments against crisis. J Mil Med. 2009;2(3):99-103.

8. Malekshahi F, Mardani M. Abilities and limitations of crisis management in Shohadaye Ashayer and Social Security hospitals of Khorramabad in 2007. Iran J Crit Care Nurs. 2009;1(1):29-34.

9. Lin C, Peng Y, Wu Y, Chang J, Chan C, Yang D. The psychological effect of severe acute respiratory syndrome on emergency department staff. Emerg Med J. 2007;24(1):12-7. doi: 10.1136/emj.2006.035089. [PubMed: 17183035].

10. Lehmann M, Bruenahl CA, Löwe B, Addo MM, Schmiedel S, Lohse AW, et al. Ebola and psychological stress of health care professionals. Emerg Infect Dis. 2015;21(5):913. doi: 10.3201/eid2105.141988. [PubMed: 25897490].

11. Folkman S, Lazarus RS, Pimley S, Novacek J. Age differences in stress and coping processes. Psychol Aging. 1987;2(2):171-84. doi: 10.1037//0882-7974.2.2.171. [PubMed: 3268206]

12. Braun V, Clarke V. Using thematic analysis in psychology. Qualit Res Psychol. 2006;3(2):77-101. doi: 10.1191/147808 8706qp063oa.

13. Schwandt TA, Lincoln YS, Guba EG. Judging interpretations: but is it rigorous? Trustworthiness and authenticity in naturalistic evaluation. $N$ Direct Evaluat. 2007;114:11-25.

14. Cai W, Lian B, Song X, Hou T, Deng G, Li H. A cross-sectional study on mental health among health care workers during the outbreak of Corona Virus Disease 2019. Asian J Psychiatry. 2020;51:102111. doi: 10.1016/j.ajp.2020.102111. [PubMed: 32361388].

15. Ferguson L, Cioffi J. Team nursing: experiences of nurse managers in acute care settings. Aust J Adv Nurs. 2011; 28(4):5-11.

16. Rebmann T, Mohr LB. Missouri nurses' bioterrorism preparedness. Biosecur Bioterror. 2008;6(3):243-51. doi: 10.1089/bsp.2008.0022. [PubMed: 18795834].

17. Considine J, Mitchell B. Chemical, biological and radiological incidents: preparedness and perceptions of emergency nurses. Disasters. 2009;33(3):482-97. doi: 10.1111/j.1467-7717.2008. 01084.x. [PubMed: 19178546].

18. Young CF, Persell DJ. Biological, chemical, and nuclear terrorism readiness: major concerns and preparedness of future nurses. Disaster Manag Response. 2004;2(4):109-14. doi: 10.1016/j.dmr.2004.08.010. [PubMed: 15448625].

19. Lam KK, Hung SYM. Perceptions of emergency nurses during the human swine influenza outbreak: a qualitative study. Int Emerg Nurs. 2013;21(4):240-6. doi: 10.1016/j.ienj.2012. 08.008. [PubMed: 23142054].

20. McMullan C, Brown GD, O'Sullivan D. Preparing to respond: Irish nurses' perceptions of preparedness for an influenza pandemic. Int Emerg Nurs. 2016;26:3-7. doi: 10.1016/j. ienj.2015.10.004. [PubMed: 26597971]. 